\title{
ON COMPACTLY SUPPORTED SPLINE WAVELETS AND A DUALITY PRINCIPLE
}

\author{
CHARLES K. CHUI AND JIAN-ZHONG WANG
}

\begin{abstract}
Let $\cdots \subset V_{-1} \subset V_{0} \subset V_{1} \subset \cdots$ be a multiresolution analysis of $L^{2}$ generated by the $m$ th order $B$-spline $N_{m}(x)$. In this paper, we exhibit a compactly supported basic wavelet $\psi_{m}(x)$ that generates the corresponding orthogonal complementary wavelet subspaces $\ldots, W_{-1}, W_{0}, W_{1}, \ldots$. Consequently, the two finite sequences that describe the two-scale relations of $N_{m}(x)$ and $\psi_{m}(x)$ in terms of $N_{m}(2 x-j), j \in \mathbb{Z}$, yield an efficient reconstruction algorithm. To give an efficient wavelet decomposition algorithm based on these two finite sequences, we derive a duality principle, which also happens to yield the dual bases $\left\{\widetilde{N}_{m}(x-j)\right\}$ and $\left\{\widetilde{\psi}_{m}(x-j)\right\}$, relative to $\left\{N_{m}(x-j)\right\}$ and $\left\{\psi_{m}(x-j)\right\}$, respectively.
\end{abstract}

\section{INTRODUCTION}

Let $m$ be any positive integer and let $N_{m}$ denote the $m$ th order $B$-spline with knots at the set $\mathbb{Z}$ of integers, such that

$$
\operatorname{supp}\left(N_{m}\right)=[0, m] \text {. }
$$

More precisely, $N_{m}$ is defined recursively by

$$
N_{m}(x)=\left(N_{m-1} * N_{1}\right)(x)=\int_{0}^{1} N_{m-1}(x-t) d t
$$

with $N_{1}=\chi_{[0,1)}$. For any $k, j \in \mathbb{Z}$, we set

$$
N_{m ; k, j}(x)=N_{m}\left(2^{k} x-j\right) ;
$$

and for each $k$, let $V_{k}$ denote the $L^{2}$-closure of the algebraic linear span

$$
\left\langle N_{m ; k, j}: j \in \mathbb{Z}\right\rangle \text {. }
$$

Here and throughout, $L^{2}=L^{2}(\mathbb{R})$. It is well known (cf. [4]) that these spline spaces $V_{k}, k \in \mathbb{Z}$, constitute a multiresolution analysis of $L^{2}$ in the sense that

(i) $\cdots \subset V_{-1} \subset V_{0} \subset V_{1} \subset \cdots$;

Received by the editors May 25, 1990 .

1980 Mathematics Subject Classification (1985 Revision). Primary 41A15, 42C05; Secondary $41 \mathrm{~A} 05,41 \mathrm{~A} 30$.

Key words and phrases. Compactly supported wavelets, $B$-splines, decomposition, reconstruction, duality principle, dual bases.

Supported by NSF Grants DMS 89-0-01345 and INT-87-12424 and SDIO/IST managed by the U.S. Army Research Office under Contract No. DAAL 03-90-G-0091. 
(ii) $\operatorname{clos}_{L^{2}}\left(\bigcup_{k \in \mathbb{Z}} V_{k}\right)=L^{2}$;

(iii) $\bigcap_{k \in \mathbb{Z}} V_{k}=\{0\}$; and

(iv) for each $k,\left\{N_{m ; k, j}: j \in \mathbb{Z}\right\}$ is an unconditional basis of $V_{k}$.

Following Mallat [4], we consider the orthogonal complementary subspaces $\ldots, W_{-1}, W_{0}, W_{1}, \ldots$; that is,

(v) $V_{k+1}=V_{k} \oplus W_{k}$, all $k \in \mathbb{Z}$,

where the notation $\oplus$ stands for $V_{k} \perp W_{k}$ and $V_{k+1}=V_{k}+W_{k}$. A trivial consequence of $(\mathrm{v})$ is that

(vi) $W_{k} \perp W_{j}$, all $k \neq j$;

and in view of (i), (ii), (iii), and (vi), it is also easy to see that

(vii) $L^{2}=\bigoplus_{k \in \mathbb{Z}} W_{k}$.

These subspaces $W_{k}, k \in \mathbb{Z}$, are called the wavelet subspaces of $L^{2}$ relative to the $B$-spline $N_{m}$. The importance of the approximation properties (ii)(iii) and the wavelet properties (v)-(vi) is that every function $f$ in $L^{2}$ can be approximated as close as we wish by some $f_{k} \in V_{k}$, for a sufficiently large value of $k$, and that $f_{k}$ has a (unique) orthogonal decomposition

$$
f_{k}=g_{k-1}+\cdots+g_{k-l}+f_{k-l},
$$

where $g_{j} \in W_{j}, j=k-l, \ldots, k-1$, and $f_{k-l} \in V_{k-l}$, and where $l$ is an arbitrarily large positive integer, so chosen that $\left\|f_{k-l}\right\|_{2}$ is as small as we desire. We call (1.2) a wavelet decomposition of $f_{k}$.

It is well known that the wavelet subspaces $W_{k}, k \in \mathbb{Z}$, are also generated by some basic wavelet in the same manner as that the spline subspaces $V_{k}, k \in \mathbb{Z}$, are generated by the $B$-spline $N_{m}$. A standard technique to determine a basic wavelet can be briefly summarized as follows (cf. [3, 4]). First, orthonormalize $\left\{N_{m ; 0, j}\right\}$, yielding $\left\{\check{N}_{m ; 0, j}\right\}$; then find the two-scale relation of $\check{N}_{m ; 0,0}$ in terms of $\left\{\tilde{N}_{m ; 1, j}\right\}$; and finally alternate the signs of the coefficient sequence in this two-scale relation in a clever way to yield the desired basic wavelet. In our earlier work [2], we introduced a different approach and showed that the $m$ th order spline function

$$
\eta_{m}(x):=L_{2 m}^{(m)}(2 x-1)
$$

also generates $W_{0}$, and consequently all the wavelet subspaces $W_{k}, k \in \mathbb{Z}$. Here and throughout, $L_{2 m}$ denotes the $(2 m)$ th order spline with knots at $\mathbb{Z}$ that satisfies the interpolatory conditions

$$
L_{2 m}(n)=\delta_{n, 0}, \quad n \in \mathbb{Z} .
$$

In the spline literature [5], $L_{2 m}$ is called the fundamental spline of order $2 m$. Let us consider the $B$-spline series representation

$$
L_{2 m}(x)=\sum_{j \in \mathbb{Z}} \alpha_{j}^{(m)} N_{2 m}(x+m-j)
$$

and denote by

$$
A(z)=\sum_{j \in \mathbb{Z}} \alpha_{j}^{(m)} z^{j}
$$


the symbol of the coefficient sequence. Then the interpolatory condition of $L_{2 m}$ at $\mathbb{Z}$ is equivalent to the identity

$$
A(z) B(z)=1
$$

where

$$
B(z)=\sum_{j=-m+1}^{m-1} N_{2 m}(m+j) z^{j}
$$

is the $B$-spline symbol and is related to the well-known Euler-Frobenius polynomial $\Pi_{m}(z)$, of degree $2 m-2$, by the identity

$$
B(z)=\frac{1}{(2 m-1) !} z^{-m+1} \Pi_{m}(z) .
$$

For more details, see Schoenberg [5] and our previous work [2].

The basic wavelet $\eta_{m}$ in (1.3) which was introduced in [2] (where the notation $\psi$ is used) has exponential decay. In fact the exact exponent is given by the magnitude of the closest root of $\Pi_{m}$ to -1 (cf. [5, pp. 37-38] and [2, (2.24)]).

In this paper, we give another basic wavelet $\psi_{m}$ which has compact support and generates $W_{0}$, and consequently all the wavelet subspaces $W_{k}, k \in \mathbb{Z}$.

The exact formula of our $\psi_{m}$ will be given in the next section, where the two-scale relation of $\psi_{m}(x)$ in terms of $N_{m}(2 x-j), j \in \mathbb{Z}$, is also derived. Hence, as in [2], together with the well-known two-scale relation:

$$
N_{m}(x)=\sum_{j=0}^{m} 2^{-m+1}\left(\begin{array}{c}
m \\
j
\end{array}\right) N_{m}(2 x-j)
$$

we have two finite sequences that yield a very efficient algorithm for reconstructing $f_{k}$ in (1.2) from its orthogonal components $g_{k-1}, \ldots, g_{k-l}, f_{k-l}$ which may have been "modified" (or "filtered" in such applications as signal processing). To prove that $\psi_{m}$ generates all of $W_{0}$, we even derive the decomposition formula of $N_{m}(2 x-j)$ in terms of $N_{m}(x-l)$ and $\psi_{m}(x-l), l \in \mathbb{Z}$, so that as in [2] again, we have an algorithm for decomposing $f_{k}$ in (1.2) into the sum of its orthogonal components $g_{k-1}, \ldots, g_{k-l}, f_{k-l}$.

A duality principle which essentially states that the pair of two-scale relations can be used as the decomposition formula, and vice versa, will be introduced in $\S 3$, where the solutions $N_{m}^{*}$ and $\psi_{m}^{*}$ of the new (i.e. dual) two-scale relations are determined. It will be shown that $N_{m}^{*}$ generates the multiresolution spaces $\left\{V_{n}\right\}$, while $\psi_{m}^{*}$ generates the wavelets spaces $\left\{W_{n}\right\}$. Hence, by using the spline $N_{m}^{*}$ and basic wavelet $\psi_{m}^{*}$, we have two finite sequences that yield a very efficient decomposition algorithm. In addition, it so happens that $\left\{N_{m}^{*}(x-j)\right\}$ gives rise to the dual basis of $\left\{N_{m}(x-j)\right\}$, and $\left\{\psi_{m}^{*}(x-j)\right\}$ yields the dual basis of $\left\{\psi_{m}(x-j)\right\}$. Consequently, every $L^{2}$ function can be easily decomposed as a direct sum of the compactly supported spline wavelets $\psi_{m ; k, j}$ which have very simple explicit formulations.

\section{COMPACTLY SUPPORTED SPLINE WAVELETS}

Let $m$ be an arbitrary positive integer which will be fixed throughout this paper, and let $N_{m}$ denote the $m$ th order $B$-spline defined in (1.1) that generates the multiresolution analysis (i)-(iv). We have the following result. 
Theorem 1. The mth order spline

$$
\psi_{m}(x)=\frac{1}{2^{m-1}} \sum_{j=0}^{2 m-2}(-1)^{j} N_{2 m}(j+1) N_{2 m}^{(m)}(2 x-j),
$$

with support $[0,2 m-1]$, is a basic wavelet that generates $W_{0}$, and consequently, all the wavelet spaces $W_{k}, k \in \mathbb{Z}$.

We remark that

$$
\psi_{1}(x)= \begin{cases}1 & \text { for } 0<x<\frac{1}{2} \\ -1 & \text { for } \frac{1}{2}<x<1 \\ 0 & \text { otherwise }\end{cases}
$$

is the well-known Haar function.

Proof. It is clear that

$$
\text { supp } \psi_{m}=[0,2 m-1] \text {. }
$$

We first show that $\psi_{m}(x-j)$ is orthogonal to $V_{0}$. To do so, we recall that

$$
N_{m}^{(m)}(x)=\sum_{j=0}^{m}(-1)^{j}\left(\begin{array}{c}
m \\
j
\end{array}\right) \delta(x-j)
$$

(cf. [1, Chapter 1]), where $\delta$ denotes, as usual, the Dirac delta distribution. Hence, by setting $k=l_{2}-l_{1}$, we have

$$
\begin{aligned}
\int_{-\infty}^{\infty} & \psi_{m}\left(x-l_{1}\right) N_{m}\left(x-l_{2}\right) d x \\
= & \sum_{j \in \mathbb{Z}} \frac{(-1)^{j}}{2^{m-1}} \cdot N_{2 m}(j+1) \int_{-\infty}^{\infty} N_{2 m}^{(m)}(2 x-j) N_{m}(x-k) d x \\
= & \sum_{j \in \mathbb{Z}} \frac{(-1)^{m+j}}{2^{2 m-1}} N_{2 m}(j+1) \int_{-\infty}^{\infty} N_{2 m}(2 x-j+2 k) N_{m}^{(m)}(x) d x \\
= & \sum_{p=0}^{m} \frac{(-1)^{m+p}}{2^{2 m-1}}\left(\begin{array}{c}
m \\
p
\end{array}\right) \sum_{j \in \mathbb{Z}}(-1)^{j} N_{2 m}(j+1) N_{2 m}(2 p+2 k-j)=0
\end{aligned}
$$

for all $k$, or all $l_{1}$ and $l_{2}$, since the positive and negative terms exactly cancel one another as follows:

$$
\begin{aligned}
\sum_{j \in \mathbb{Z}}(-1)^{j} & N_{2 m}(j+1) N_{2 m}(2 p+2 k-j) \\
= & \sum_{j \in \mathbb{Z}} N_{2 m}(2 j+1) N_{2 m}(2 p+2 k-2 j) \\
& -\sum_{j \in \mathbb{Z}} N_{2 m}(2 j) N_{2 m}(2 p+2 k-2 j+1)=0 .
\end{aligned}
$$

To verify that $\psi_{m}$ is in $V_{1}$, we need the spline identity

$$
N_{2 m}^{(m)}(x)=\sum_{j=0}^{m}(-1)^{j}\left(\begin{array}{c}
m \\
j
\end{array}\right) N_{m}(x-j)
$$


(cf. [1, Chapter 1]). So, putting (2.2) into (2.1), we arrive at the two-scale relation:

$$
\psi_{m}(x)=\sum_{n=0}^{3 m-2} q_{n} N_{m}(2 x-n)
$$

where

$$
q_{n}=\frac{(-1)^{n}}{2^{m-1}} \sum_{j=0}^{m}\left(\begin{array}{c}
m \\
j
\end{array}\right) N_{2 m}(n-j+1) .
$$

Finally, to show that $\psi_{m}$ generates all of $W_{0}$, we derive a decomposition relation; that is, we determine two $l^{2}$ sequences $\left\{a_{n}\right\}$ and $\left\{b_{n}\right\}$ such that

$$
N_{m}(2 x-l)=\sum_{n \in \mathbb{Z}} a_{l-2 n} N_{m}(x-n)+\sum_{n \in \mathbb{Z}} b_{l-2 n} \psi_{m}(x-n)
$$

for all $l \in \mathbb{Z}$. These two sequences have to depend on the sequences $\left\{p_{n}\right\}$ and $\left\{q_{n}\right\}$ that define the pair of two-scale relations (1.9) and (2.3), where the notation

$$
p_{n}= \begin{cases}2^{-m+1}\left(\begin{array}{c}
m \\
n
\end{array}\right) & \text { for } 0 \leq n \leq m \\
0 & \text { otherwise }\end{cases}
$$

for the coefficients in (1.9) is used. Of course, it follows from (2.4) that $q_{n}=0$ for $n<0$ or $n>3 m-2$. To determine $\left\{a_{n}\right\}$ and $\left\{b_{n}\right\}$ in (2.5), it is more convenient to use Fourier transform representations. To do so, we need the notations:

$$
\begin{cases}P(z)=\sum_{n=0}^{m} p_{n} z^{n}, & Q(z)=\sum_{n=0}^{3 m-2} q_{n} z^{n}, \\ G(z)=\sum_{n \in \mathbb{Z}} a_{n} z^{-n}, & H(z)=\sum_{n \in \mathbb{Z}} b_{n} z^{-n} .\end{cases}
$$

Hence, by setting

$$
z=e^{-i \frac{\omega}{2}}
$$

the Fourier transform representations of the identities (1.9), (2.3), and (2.5) become

$$
\begin{aligned}
\widehat{N}_{m}(\omega) & =\frac{1}{2} P(z) \widehat{N}_{m}\left(\frac{\omega}{2}\right), \\
\widehat{\psi}_{m}(\omega) & =\frac{1}{2} Q(z) \widehat{N}_{m}\left(\frac{\omega}{2}\right),
\end{aligned}
$$

and

$$
\left\{\begin{array}{l}
\frac{1}{2} \widehat{N}_{m}\left(\frac{\omega}{2}\right)=\frac{G(z)+G(-z)}{2} \widehat{N}_{m}(\omega)+\frac{H(z)+H(-z)}{2} \widehat{\psi}_{m}(\omega), \\
\frac{1}{2} \widehat{N}_{m}\left(\frac{\omega}{2}\right)=\frac{G(z)-G(-z)}{2} \widehat{N}_{m}(\omega)+\frac{H(z)-H(-z)}{2} \widehat{\psi}_{m}(\omega),
\end{array}\right.
$$

respectively, where the first identity in $(2.11)$ is the Fourier transform representation of the identity (2.5) for even $l$, and the second identity in (2.11) 
corresponds to odd values of $l$ in (2.5). By using (2.9) and (2.10), the pair of identities in $(2.11)$ can be written as

$$
\left\{\begin{array}{l}
P(z) G(z)+Q(z) H(z)=2 \\
P(z) G(-z)+Q(z) H(-z)=0
\end{array}\right.
$$

Hence, solving for $\left\{a_{n}\right\}$ and $\left\{b_{n}\right\}$ in the decomposition relation (2.5) is equivalent to solving for $G(z)$ and $H(z)$ in (2.12). From (2.6) and (2.4), it is clear that

$$
\begin{aligned}
& P(z)=2^{-m+1}(1+z)^{m} \quad \text { and } \\
& Q(z)=\frac{2^{-m+1}}{(2 m-1) !}(1-z)^{m} \Pi_{m}(-z),
\end{aligned}
$$

which are polynomials of degrees $m$ and $3 m-2$, respectively. The following identity for the Euler-Frobenius polynomials $\Pi_{m}$ of degree $2 m-2$, which was derived in [2], facilitates our solution for $G(z)$ and $H(z)$ :

$$
\frac{(1+z)^{2 m} \Pi_{m}(z)}{z \Pi_{m}\left(z^{2}\right)}-\frac{(1-z)^{2 m} \Pi_{m}(-z)}{z \Pi_{m}\left(z^{2}\right)}=2^{2 m} .
$$

Indeed, with the aid of (2.15), it follows from (2.13) and (2.14), that

$$
G(z)=\frac{1}{2^{m}}(1+z)^{m} \frac{\Pi_{m}(z)}{z \Pi_{m}\left(z^{2}\right)}
$$

and

$$
H(z)=\frac{-(2 m-1) !}{2^{m}}(1-z)^{m} \frac{1}{z \Pi_{m}\left(z^{2}\right)} .
$$

Recall from (1.6), (1.8), and (1.5) that

$$
\frac{1}{z \Pi_{m}\left(z^{2}\right)}=\frac{z^{-2 m+1}}{(2 m-1) !} A\left(z^{2}\right)=\frac{1}{(2 m-1) !} \sum_{n \in \mathbb{Z}} \alpha_{n}^{(m)} z^{2 n-2 m+1} .
$$

Hence, in view of (2.16) and (2.17), the decomposition sequences $\left\{a_{n}\right\}$ and $\left\{b_{n}\right\}$ in (2.5) and (2.7) are simply (finite) linear combinations of the sequence $\left\{\alpha_{n}^{(m)}\right\}$ which defines the fundamental cardinal spline $L_{2 m}$ in (1.4). This completes the proof of Theorem 1 .

Recall from [5, pp. 37-38] that the rate of decay of $\left\{\alpha_{n}^{(m)}\right\}$ is $O\left(\left|r_{m}\right|^{-|n|}\right)$ as $|n| \rightarrow \infty$, where $r_{1}, \ldots, r_{2 m-2}$ are the roots of $\Pi_{m}(z)$ labeled in decreasing order:

$$
r_{2 m-2}<r_{2 m-3}<\cdots<r_{m}<-1<r_{m-1}<\cdots<r_{1}<0 .
$$

Hence, we have

$$
a_{n}, b_{n}=O\left(\left|r_{m}\right|^{-\frac{|n|}{2}}\right), \quad|n| \rightarrow \infty .
$$

Following our earlier work [2], we see that the two-scale (finite) sequences $\left\{p_{n}\right\}$ and $\left\{q_{n}\right\}$ yield a very efficient reconstruction algorithm, while the decomposition (exponential decay) sequences $\left\{a_{n}\right\}$ and $\left\{b_{n}\right\}$ define a decomposition algorithm. Since decomposition is usually more delicate than reconstruction, it is important to have finite sequences with very small supports for the decomposition algorithm while maintaining the certain order of smoothness of the 
"spline" and "wavelet" functions such as our $N_{m}$ and $\psi_{m}$ which are both in $C^{m-2}$. A duality principle will be introduced in the next section to transform the finite two-scale sequences $\left\{p_{n}\right\}$ and $\left\{q_{n}\right\}$ into the desired pair of decomposition sequences without leaving the spline and wavelet spaces $\left\{V_{n}\right\}$ and $\left\{W_{n}\right\}$. We remark that Daubechies' compactly supported (nonspline) wavelets [3] certainly yield finitely supported decomposition and reconstruction sequences, but the supports of these sequences have to be quite large if a certain order of smoothness (or regularity) is desired (cf. [3] for details). We will also see that since our compactly supported spline wavelet $\psi_{m}$ has a very simple explicit formulation, this duality yields a very desirable decomposition of every $L^{2}$ function into a direct sum of the wavelets $\psi_{m ; k, j}$.

\section{A DUALITY PRINCIPLE AND DUAL BASES}

As mentioned above, in this section we attempt to interchange the pair $\left(\left\{p_{n}\right\},\left\{q_{n}\right\}\right)$ of two-scale sequences with the pair $\left(\left\{a_{n}\right\},\left\{b_{n}\right\}\right)$ of decomposition sequences. However, due to the two-scale property, we find it more convenient to carry a factor of 2 . In other words, let us define

$$
\left\{\begin{array}{l}
P^{*}(z)=2 G(z)=\sum_{n \in \mathbb{Z}} p_{n}^{*} z^{n}, \\
Q^{*}(z)=2 H(z)=\sum_{n \in \mathbb{Z}} q_{n}^{*} z^{n}, \\
G^{*}(z)=\frac{1}{2} P(z)=\sum_{n=0}^{m} a_{-n}^{*} z^{n} \\
H^{*}(z)=\frac{1}{2} Q(z)=\sum_{n=0}^{3 m-2} b_{-n}^{*} z^{n}
\end{array}\right.
$$

which is equivalent to setting

$$
p_{n}^{*}=2 a_{-n}, \quad q_{n}^{*}=2 b_{-n}, \quad a_{-n}^{*}=\frac{1}{2} p_{n}, \quad b_{-n}^{*}=\frac{1}{2} q_{n},
$$

where the sequences $\left\{a_{n}\right\},\left\{b_{n}\right\},\left\{p_{n}\right\}$, and $\left\{q_{n}\right\}$ were defined in the previous section. It is clear from the definitions (3.1) and (3.2) and the identities in (2.12), that provided that the pair of two-scale relations

$$
\widehat{N}_{m}^{*}(\omega)=\frac{1}{2} P^{*}(z) \widehat{N}_{m}^{*}\left(\frac{\omega}{2}\right)
$$

and

$$
\widehat{\psi}_{m}^{*}(\omega)=\frac{1}{2} Q^{*}(z) \widehat{N}_{m}^{*}\left(\frac{\omega}{2}\right)
$$

where $z=e^{-i \frac{\omega}{2}}$, have solutions $N_{m}^{*}$ and $\psi_{m}^{*}$ that generate $\left\{V_{n}\right\}$ and $\left\{W_{n}\right\}$, respectively, the decomposition relation

$$
N_{m}^{*}(2 x-l)=\sum_{n} \frac{1}{2} p_{2 n-l} N_{m}^{*}(x-n)+\sum_{n} \frac{1}{2} q_{2 n-l} \psi_{m}^{*}(x-n)
$$


is automatically satisfied. So, it is sufficient to study the relations in (3.3a) and (3.3b), or equivalently, the relations

$$
N_{m}^{*}(x)=\sum_{n} 2 a_{n} N_{m}^{*}(2 x+n)
$$

and

$$
\psi_{m}^{*}(x)=\sum_{n} 2 b_{n} N_{m}^{*}(2 x+n)
$$

To state the following result, we need the basic wavelet $\eta_{m}$ in (1.3) which was introduced in our previous work [2] (where the notation $\psi$ is used).

Theorem 2. The mth order spline

$$
N_{m}^{*}(x):=\sum_{j \in \mathbb{Z}} \alpha_{j}^{(m)} N_{m}(x+m-j)
$$

generates $V_{0}$ and satisfies the two-scale relation (3.5a), and the (spline) wavelet

$$
\psi_{m}^{*}(x):=-2^{-m+1} \sum_{j \in \mathbb{Z}} \alpha_{j}^{(m)} \eta_{m}(x+m-j)
$$

generates $W_{0}$ and satisfies the two-scale relation (3.5b).

Proof. It is clear that $N_{m}^{*} \in V_{0}$ and $\psi_{m}^{*} \in W_{0}$. By using the interpolatory property $L_{2 m}(k)=\delta_{k, 0}$, we also have, from (1.4),

$$
\sum_{j \in \mathbb{Z}} \alpha_{j}^{(m)} N_{2 m}(k+m-j)=\delta_{k, 0} .
$$

Hence, it follows from (3.6) and (3.8) that

$$
\begin{aligned}
\sum_{l} N_{2 m}(l) N_{m}^{*}(x-l) & =\sum_{l} \sum_{j} \alpha_{j}^{(m)} N_{2 m}(l) N_{m}(x+m-j-l) \\
& =\sum_{k}\left\{\sum_{j} \alpha_{j}^{(m)} N_{2 m}(k+m-j)\right\} N_{m}(x-k) \\
& =\sum_{k} \delta_{k, 0} N_{m}(x-k)=N_{m}(x),
\end{aligned}
$$

so that $N_{m}^{*}$ generates $V_{0}$. To verify that $N_{m}^{*}$ satisfies the two-scale relation (3.5a), it is equivalent to verifying $(3.3 \mathrm{a})$, or

$$
\widehat{N}_{m}^{*}(\omega)=G(z) \widehat{N}_{m}^{*}\left(\frac{\omega}{2}\right)
$$

This is certainly true since from (3.6), we have

$$
\widehat{N}_{m}^{*}(\omega)=z^{-2 m} A\left(z^{2}\right) \widehat{N}_{m}(\omega),
$$

so that it follows from (1.6), (1.8), and (2.16) that

$$
\begin{aligned}
\frac{\widehat{N}_{m}^{*}(\omega)}{\widehat{N}_{m}^{*}\left(\frac{\omega}{2}\right)} & =\frac{1}{z^{m}} \cdot \frac{A\left(z^{2}\right)}{A(z)} \cdot \frac{\widehat{N}_{m}(\omega)}{\widehat{N}_{m}\left(\frac{\omega}{2}\right)}=\frac{1}{2 z^{m}} P(z) \frac{A\left(z^{2}\right)}{A(z)} \\
& =\frac{1}{2^{m} z^{m}}(1+z)^{m} \frac{z^{-m+1} \Pi_{m}(z)}{z^{-2 m+2} \Pi_{m}\left(z^{2}\right)}=G(z) .
\end{aligned}
$$


Similarly, as in (3.9), it follows from (3.7) and (3.8) that

$$
\begin{aligned}
\sum_{l} & -2^{m-1} N_{2 m}(l) \psi_{m}^{*}(x-l) \\
& =\sum_{l} \sum_{j} \alpha_{j}^{(m)} N_{2 m}(l) \eta_{m}(x+m-j-l)=\eta_{m}(x) .
\end{aligned}
$$

Since $\eta_{m}$ generates $W_{0}$ (cf. [2]), we conclude that $\psi_{m}^{*}$ also generates $W_{0}$. To verify the two-scale relation (3.5b), it is equivalent to verifying its Fourier transform representation $(3.3 \mathrm{~b})$, or

$$
\widehat{\psi}_{m}^{*}(\omega)=H(z) \widehat{N}_{m}^{*}\left(\frac{\omega}{2}\right) .
$$

This can be seen by applying (2.15), (3.7) and (3.10). Indeed,

$$
\begin{aligned}
\frac{\widehat{\psi}_{m}^{*}(\omega)}{\widehat{N}^{*}\left(\frac{\omega}{2}\right)} & =-2^{-m+1} z^{-2 m} A\left(z^{2}\right) \hat{\eta}_{m}(z) \frac{1}{z^{-m} A(z) \widehat{N}\left(\frac{\omega}{2}\right)} \\
& =-\frac{A\left(z^{2}\right)\left[\frac{1}{2}(1-z)^{m} z^{-m+1}\right] A(z) \widehat{N}\left(\frac{\omega}{2}\right)}{2^{m-1} z^{m} A(z) \widehat{N}\left(\frac{\omega}{2}\right)} \\
& =-\frac{(1-z)^{m}}{2^{m} z^{2 m-1}} A\left(z^{2}\right)=\frac{-(2 m-1) !}{2^{m}} \frac{(1-z)^{m}}{z \Pi_{m}\left(z^{2}\right)} \\
& =H(z) .
\end{aligned}
$$

This completes the proof of the theorem.

It turns out that this duality principle yields the dual bases of the $B$-spline basis $\left\{N_{m}(x-j): j \in \mathbb{Z}\right\}$ and the basic wavelet basis $\left\{\psi_{m}(x-j): j \in \mathbb{Z}\right\}$. For this purpose, we have the following result.

Theorem 3. For all $j, k \in \mathbb{Z}$,

$$
\int_{-\infty}^{\infty} N_{m}(x+m-j) N_{m}^{*}(x-k) d x=\delta_{j, k}
$$

and

$$
(-1)^{m} \int_{-\infty}^{\infty} \psi_{m}(x+2 m-1-j) \psi_{m}^{*}(x-k) d x=\delta_{j, k} .
$$

Proof. The proof of (3.11) depends on the Poisson summation formula which yields

$$
\begin{aligned}
\sum_{j \in \mathbb{Z}} \widehat{N}_{2 m}(\omega+2 \pi j) & =\sum_{j \in \mathbb{Z}} N_{2 m}(j) e^{-i j \omega} \\
& =z^{2 m} \sum_{j \in \mathbb{Z}} N_{2 m}(m+j) z^{2 j}=\frac{z^{2 m}}{A\left(z^{2}\right)}
\end{aligned}
$$

where $z=e^{-i \frac{\omega}{2}}$ and the last equality is a consequence of (1.7) and (1.6). 
Hence, by applying (3.10) and (3.13), we have

$$
\begin{aligned}
\int_{-\infty}^{\infty} & N_{m}^{*}(x) N_{m}(x+m-k) d x \\
& =\frac{1}{2 \pi} \int_{-\infty}^{\infty} \widehat{N}_{m}^{*}(\omega) \overline{\widehat{N}_{m}(\omega) e^{i(m-k) \omega}} d \omega \\
& =\frac{1}{2 \pi} \int_{-\infty}^{\infty} z^{-2 m} A\left(z^{2}\right)\left(\widehat{N}_{m}(\omega)\right)^{2} e^{i k \omega} d \omega \\
& =\frac{1}{2 \pi} \int_{-\infty}^{\infty} z^{-2 m} A\left(z^{2}\right) \widehat{N}_{2 m}(\omega) e^{i k \omega} d \omega \\
& =\frac{1}{2 \pi} \sum_{j \in \mathbb{Z}} \int_{2 \pi j}^{2 \pi(j+1)} z^{-2 m} A\left(z^{2}\right) \widehat{N}_{2 m}(\omega) e^{i k \omega} d \omega \\
& =\frac{1}{2 \pi} \int_{0}^{2 \pi} z^{-2 m} A\left(z^{2}\right) e^{i k \omega}\left(\sum_{j \in \mathbb{Z}} \widehat{N}_{2 m}(\omega+2 \pi j)\right) d \omega \\
& =\frac{1}{2 \pi} \int_{0}^{2 \pi} e^{i k \omega} d \omega=\delta_{k, 0} .
\end{aligned}
$$

This verifies (3.11). To establish (3.12), we first observe that by applying the two-scale relations to $(3.14)$, we obtain

$$
\begin{aligned}
\delta_{k, 0} & =\frac{1}{2 \pi} \int_{-\infty}^{\infty}\left(\frac{1}{2} \overline{P(z)}\right)\left(\frac{1}{2} P^{*}(z)\right) \widehat{N}_{m}^{*}\left(\frac{\omega}{2}\right) \overline{\hat{N}_{m}\left(\frac{\omega}{2}\right)} e^{-i(m-k) \omega} d \omega \\
& =\frac{1}{4 \pi} \int_{-\infty}^{\infty} z^{m} P(z) G(z) \widehat{N}_{m}^{*}\left(\frac{\omega}{2}\right) \overline{\widehat{N}_{m}\left(\frac{\omega}{2}\right)} e^{i k \omega} d \omega .
\end{aligned}
$$

Hence, by applying the other pair of two-scale relations, the first identity in (2.12), and (3.14) again, we arrive at

$$
\begin{aligned}
& \int_{-\infty}^{\infty} \psi_{m}^{*}(x) \psi_{m}(x+2 m-1-k) d x \\
&=\frac{1}{2 \pi} \int_{-\infty}^{\infty} \widehat{\psi}_{m}^{*}(\omega) \overline{\hat{\psi}_{m}(\omega)} e^{-i(2 m-1-k) \omega} d \omega \\
&= \frac{1}{2 \pi} \int_{-\infty}^{\infty}\left(\frac{1}{2} \overline{Q(z)}\right)\left(\frac{1}{2} Q^{*}(z)\right) \widehat{N}_{m}^{*}\left(\frac{\omega}{2}\right) \overline{\widehat{N}_{m}\left(\frac{\omega}{2}\right)} e^{-i(2 m-1-k) \omega} d \omega \\
&= \frac{(-1)^{m}}{4 \pi} \int_{-\infty}^{\infty} z^{m} Q(z) H(z) \widehat{N}_{m}^{*}\left(\frac{\omega}{2}\right) \overline{\widehat{N}_{m}\left(\frac{\omega}{2}\right)} e^{i k \omega} d \omega \\
&= \frac{(-1)^{m}}{2 \pi} \int_{-\infty}^{\infty} \widehat{N}_{m}^{*}\left(\frac{\omega}{2}\right) \overline{\widehat{N}_{m}\left(\frac{\omega}{2}\right)} e^{i\left(k-\frac{m}{2}\right) \omega} d \omega \\
&-\frac{(-1)^{m}}{4 \pi} \int_{-\infty}^{\infty} z^{m} P(z) G(z) \widehat{N}_{m}^{*}\left(\frac{\omega}{2}\right) \widehat{N}_{m}\left(\frac{\omega}{2}\right) e^{i k \omega} d \omega \\
&=(-1)^{m}\left(2 \delta_{2 k, 0}-\delta_{k, 0}\right)=(-1)^{m} \delta_{k, 0} .
\end{aligned}
$$


As a trivial consequence of Theorem 3, we can now write down the dual bases of the $B$-splines $\left\{N_{m}(x-j)\right\}$ and the compactly supported basic wavelets $\left\{\psi_{m}(x-j)\right\}$ as follows. Let

$$
\tilde{N}_{m}(x)=N_{m}^{*}(x-m)=\sum_{j \in \mathbb{Z}} \alpha_{j}^{(m)} N_{m}(x-j)
$$

and

$$
\begin{aligned}
\widetilde{\psi}_{m}(x) & =(-1)^{m} \psi_{m}^{*}(x-2 m+1) \\
& =\frac{(-1)^{m+1}}{2^{m-1}} \sum_{j \in \mathbb{Z}} \alpha_{j}^{(m)} \eta_{m}(x-m+1-j) .
\end{aligned}
$$

Then by using the standard notation

$$
\langle f, g\rangle=\int_{-\infty}^{\infty} f(x) \overline{g(x)} d x
$$

we have

$$
\left\langle N_{m}(\cdot-j), \tilde{N}_{m}(\cdot-l)\right\rangle=\delta_{j, l}
$$

and

$$
\left\langle\psi_{m}(\cdot-j), \widetilde{\psi}_{m}(\cdot-l)\right\rangle=\delta_{j, l}
$$

for all $j, l \in \mathbb{Z}$. In addition, if we set

$$
\left\{\begin{array}{l}
N_{m ; k, j}(x)=N_{m}\left(2^{k} x-j\right), \\
\psi_{m ; k, j}(x)=\psi_{m}\left(2^{k} x-j\right)
\end{array}\right.
$$

and

$$
\left\{\begin{array}{l}
\tilde{N}_{m ; k, j}(x)=\tilde{N}_{m}\left(2^{k} x-j\right), \\
\widetilde{\psi}_{m ; k, j}(x)=\widetilde{\psi}_{m}\left(2^{k} x-j\right),
\end{array}\right.
$$

then every spline function $f_{k}$ in $V_{k}, k \in \mathbb{Z}$, has the $B$-spline series representation

$$
f_{k}=2^{k} \sum_{j \in \mathbb{Z}}\left\langle f_{k}, \tilde{N}_{m ; k, j}\right\rangle N_{m ; k, j}
$$

and every $f \in L^{2}$ has the wavelet decomposition

$$
f=\sum_{j, k \in \mathbb{Z}} 2^{k}\left\langle f, \widetilde{\psi}_{m ; k, j}\right\rangle \psi_{m ; k, j}
$$

where

$$
\operatorname{supp}\left(\psi_{m ; k, j}\right)=\left[\frac{j}{2^{k}}, \frac{2 m-1+j}{2^{k}}\right] .
$$

Of course the validity of (3.22) follows from the property (vii) of the wavelet spaces $\left\{W_{k}\right\}$. It should be emphasized that the coefficients $2^{\frac{k}{2}}\left\langle f, \widetilde{\psi}_{m ; k, j}\right\rangle$ in (3.22) are the integral wavelet transforms

$$
\left(W_{\widetilde{\psi}_{m}} f\right)(b, a)=\frac{1}{\sqrt{a}} \int_{-\infty}^{\infty} f(x) \overline{\tilde{\psi}_{m}\left(\frac{x-b}{a}\right)} d x
$$


of $f$ with "window wavelet" function $\tilde{\psi}$ evaluated at the dyadic points

$$
(b, a)=\left(\frac{j}{2^{k}}, 2^{-k}\right) .
$$

Hence, the decomposition algorithm discussed in [2] can be used to efficiently compute the integral wavelet transform of $f$ at these dyadic points, and the reconstruction algorithm in [2] can be used to recover $f$ from these values of the integral wavelet transform of $f$. The only difference in applying the $B$-spline and $B$-wavelet pair $\left(N_{m}, \psi_{m}\right)$ from the orthonormal wavelets of Daubechies [3], say, is that the dual $\widetilde{\psi}_{m}$ of $\psi_{m}$ is used as the window wavelet function, while orthonormal wavelets are of course self-dual. A disadvantage in our dual approach here is that while the reconstruction sequences are finite, the decomposition sequences are infinite although they have very rapid exponential decay. An advantage of our wavelets $\psi_{m}$ and $\widetilde{\psi}_{m}$ over the Daubechies wavelets is that both $\psi_{m}$ and $\widetilde{\psi}_{m}$ are either symmetric or antisymmetric, so that the filtering process has linear phase, while the non-Haar compactly supported orthogonal wavelets do not have this property. In addition, the symmetry of these "filtering" coefficients facilitates implementation of the algorithms.

Final Remarks. Nonorthogonal compactly supported wavelets have also been studied by P. Auscher in his 1989 Doctoral Thesis at the University of ParisDauphine, without giving any explicit formulas. On the other hand, biorthogonal bases have just been constructed in June, 1990 by Cohen, Daubechies, and Feauveau, and in the 1990 Doctoral Theses of A. Cohen and of Feauveau, at the University of Paris-Dauphine and the University of Paris-Sud, respectively. These bases functions are symmetric and have compact support, but orthogonality between different scale wavelet layers is lost. Hence, the work of the compactly supported wavelets $\psi_{m}$ and their corresponding dual wavelets $\widetilde{\psi}_{m}$ in this paper can be considered as intermediate between Daubechies' compactly supported orthogonal wavelets and the biorthogonal wavelets of Cohen, Daubechies, and Feauveau. This interesting observation was pointed out by the referee, to whom we are very appreciative.

\section{ACKNOWLEDGMENT}

We are very grateful to G. Battle, A. Cohen, I. Daubechies, and S. Mallat for providing us with their preprints and reprints. The second author is a Visiting Scholar at the Center for Approximation Theory of Texas A\&M University during the period September 1989-December 1990.

\section{REFERENCES}

1. C. K. Chui, Multivariate splines, CBMS-NSF Series in Applied Math. \#54, SIAM, Philadelphia, Pa., 1988.

2. C. K. Chui and J. Z. Wang, A cardinal spline approach to wavelets, Proc. Amer. Math. Soc. (to appear).

3. I. Daubechies, Orthonormal bases of compactly supported wavelets, Comm. Pure Appl. Math. 41 (1988), 909-996. 
4. S. G. Mallat, Multiresolution approximations and wavelet orthonormal bases of $L^{2}(\mathbb{R})$, Trans. Amer. Math. Soc. 315 (1989), 69-87.

5. I. J. Schoenberg, Cardinal spline interpolation, CBMS-NSF Series in Applied Math. \#12, SIAM, Philadelphia, Pa., 1973.

Department of Mathematics, Texas A\&M University, College Station, TeXas 77843 E-mail address: E443 cc@TAMVM1

Department of Mathematics, Wuhan University, Wuhan, Hubei 430072, People's RePUBLIC OF CHINA 